\title{
Dynamics of moisture interaction with polyelectrolyte multilayers containing nanofibrillated cellulose
}

\author{
Hjalmar Granberg, Ludovic G. Coppel, Mohamed Eita, Eduardo A. de Mayolo, Hans Arwin, Lars Wågberg
}

KEYWORDS: Optics, Interference, Nanofibrillated cellulose, Layer-by-Layer, Colour

SUMMARY: Recent findings have shown that it is possible to use the Layer-by-Layer technique to create nanofibrillated cellulose / polyethyleneimine interference films whose colour change with relative humidity. This study uses different optical models to describe spectral ellipsometry measurements data of interference films and how the film properties alter in dry and humid environments. The results indicate that water condensation initially is filling the surface pores within seconds whereas relaxation of the film to adjust to the added water is a slower process that reaches a steady state after $\approx 20 \mathrm{~min}$. The maximum swelling ratio of the $\mathrm{LbL}$ films is almost independent of the number of layers within the film, but decreases considerably by crosslinking via heat treatment. The films show a distinct birefringence with optical axis perpendicular to the surface. Analysis of the moisture response with different optical models indicates that the films swell uniformly in the thickness direction with no separate water film on top. The results provide important understanding for the design of NFC based LbL films for visual moisture sensors and interactive security paper.

\begin{tabular}{l}
\hline ADDRESSES OF THE AUTHORS: Hjalmar \\
Granberg (hjalmar.granberg@innventia.com), Innventia \\
AB, Box 5604, 104 86 Stockholm, Sweden, Ludovic \\
Gustafsson Coppel (ludovic.coppel@innventia.com), \\
Mohamed Eita (eita@kth.se), Eduardo Antunez de \\
Mayolo (eduan087@student.liu.se). Hans Arwin \\
(hansa@ifm.liu.se) and Lars Wågberg \\
(wagberg@kth.se) \\
Corresponding author: Hjalmar Granberg
\end{tabular}

Since the introduction in the late 1980s, the Layer-byLayer (LbL) technique (Decher et al. 1991, Decher 1997) has initiated a large interest in the scientific community. Using the LbL technique, it is possible to construct welldefined nanometer thin layers of oppositely charged polyelectrolytes and/or colloids at any solid-liquid interface.

During the last decade, there has been a large focus on using NanoFibrillated Cellulose (NFC) (Klemm et al. 2011) in new materials. NFC emanates from the cellulose fibrils inside the wood fibre wall and the fibrils are liberated in different ways using both chemical treatment and high pressure homogenization (Klemm et al. 2011; Eichhorn et al. 2010) to separate the fibrils from each other and to stabilize them in aqueous dispersion. Depending on the preparation conditions, it is also possible to create negatively as well as positively charged NFCs with different charge densities (Klemm et al. 2011) on the nanomaterial surfaces. This naturally affects the stability of the dispersions, their self-associative properties and their interactions with other nanomaterials.
It has been shown that the build-up of the LbL structure is so regular that the deposition of each NFC layer (Wågberg et al. 2008) creates a colour change after a few bilayers of polyethyleneimine (PEI) and NFC on a silicon wafer. This opened totally new possibilities to create interactive products (Wågberg et al. 2008), such as for example moisture sensors, since the layers are sensitive to moisture and will change colour depending on how much water that is adsorbed into the LbL structure. Another very interesting application is in security paper where LbL structures are first formed and then transferred to paper by for example microcontact printing. However, in all these applications it is necessary to determine how moisture interacts with the NFCcontaining LbL films. Previous works (Wong et al. 2004; Tanchak et al. 2004; Burke, Barrett 2005; Secrist, Nolte 2011; Nolte et al. 2008; Lösche et al. 1998) on the interaction of moisture with LbL films (all without NFC) have assumed an isotropic homogenous LbL film that swells in contact with moist air and that no separate water film is condensed on top of the film. This leads to the objective of the current work: How can the optical properties of the NFC based LbL thin films be described and how do they interact with the moisture in the ambient air?

This problem is studied by monitoring differently prepared LbL films at different humidity by spectral ellipsometry.

\section{Materials}

Polyethyleneimine (PEI), $\mathrm{M}_{\mathrm{w}}=60000 \mathrm{~g} / \mathrm{mol}, 53 \%$ aqueous solution, was purchased from Acros Organics (USA) and used as received. Nanofibrillated Cellulose (NFC) was prepared at Innventia AB, Sweden, according to the method described by (Wågberg et al. 2008). Polished silicon wafers ( $\mathrm{p}$-doped with boron), with a thin oxide layer were obtained from MEMC Electronic Material, Italy.

\section{Methods}

Solutions of PEI and NFC (low charge and high charge) were prepared at the concentration $100 \mathrm{mg} / \mathrm{L}$ in ultrapure water. The $\mathrm{pH}$ of the solutions was 10 for the PEI and 7 for the high and low charge NFC. Multilayers were prepared using the layer-by-layer technique with a dipping robot from Nanostrata Inc., USA. Silicon wafers were cut to the desired dimensions $(\sim 5 \mathrm{~cm} \times 1 \mathrm{~cm})$ and cleaned with water and ethanol followed by plasma treatment (PDC-002, Harrick scientific Inc.) at 30 Watt under reduced air pressure. The cleaned Silicon substrates were dipped in solutions according to the following timing scheme: $20 \mathrm{~min}$ for the precursor layer of PEI, $15 \mathrm{~min}$ for the NFC layers, and $10 \mathrm{~min}$ for each layer of PEI. Three rinsing steps in ultrapure water for 2 min each were applied between each successive layer. Thin films 
were finally dried with nitrogen after coating and kept in Petri dishes sealed with a parafilm. Cross-linking of thin films took place by placing them in the oven at $180^{\circ} \mathrm{C}$ for 2 hours. To achieve that, each silicon substrate coated with a thin film was cut into two pieces, one was placed in the oven for cross-linking and the other was kept as reference for comparison.

The samples were characterized with a variable-angle spectroscopic ellipsometer (RC2, J.A. Woollam Co., Inc.) in the wavelength range $400-1650 \mathrm{~nm}$ at $70^{\circ}$ angle of incidence. The optical data were modeled using the software CompleteEASE from the instrument manufacturer. The dynamics of the LbL films was monitored in a closed measurement chamber with an ambient controlled by purging dry nitrogen $(0 \% \mathrm{RH})$ and then adding a humid air flow $(84-88 \% \mathrm{RH})$, filling the chamber within seconds.

The refractive index and thickness of the LbL films were evaluated in a two-layer optical model with a silicon substrate $(\mathrm{Si})$ with a native silicon oxide $(\mathrm{SiOx})$ layer with an LbL film on top. The scattering matrix algorithm (Azzam, Bashara, 1986) was used for calculation of model generated ellipsometry data with $\mathrm{Si}$ and $\mathrm{SiOx}$ refractive indices taken from the literature (Herzinger et al, 1998). The SiOx thickness was determined to $2.6 \mathrm{~nm}$ on a separate sample. The real part $n$ of the refractive index of LbL films was parameterized by a Cauchy dispersion (Azzam, Bashara, 1986) according to

$$
n(\lambda)=A+\frac{B}{\lambda^{2}}
$$

where $A$ and $B$ are fit parameters and $\lambda$ is the wavelength. In addition a so called Urbach tail (Azzam, Bashara, 1986), an imaginary part $k$ of the refractive index which decreases exponentially with $\lambda$, as well as the LbL layer thickness $t$ were fit parameters. The difference in terms of the mean square error (MSE) between model generated data and experimental data (NSC representation) were minimized by allowing the fit parameters to vary using the non-linear regression Levenberg-Marquardt algorithm (Levenberg, 1944). In the basic model described above we assume planar interfaces and isotropic materials. Model improvements were evaluated by comparing MSE among different models including: a) addition of a thin water layer $\left(n_{\text {water }} \approx 1.33\right.$ ) on top of the LbL film; b) introduction of a thickness variation of the LbL film, a so called thickness inhomogeneity with a Gaussian distribution of thicknesses, assuming nine different thickness values centered around an average and with the Gaussian width and the center average thickness as fit parameters; c) using uniaxial birefringence of the LbL film with the optical axis normal to the surface and with Cauchy dispersions $(E q 1)$ for the in-plane as well as outof-plane refractive indices, defined by different $A$-values; and d) a linear grading of the Cauchy index of the LbL film normal to the surface.

\section{Results and discussion}

All samples exhibited interference colours. The LbL films also showed a colour change when exposed to the saturated water vapor in exhaled air from a human observer.

Fig 1 (left) shows the relative thickness (swelling and deswelling) of the LbL films when exposed to different relative humidity $(\mathrm{RH})$. The curves show pairwise similar swelling within $5 \%$, i.e. pair (1) noncrosslinked 4 and 48 bilayers of PEI / high charged NFC (black curves), pair (2) crosslinked 4 and 48 bilayers of PEI / high charged NFC (blue curves), and pair (3) 5 and 15 layers of PEI / low charge NFC (gray and red curves). Hence it can be concluded that the swelling is almost independent of the number of bilayers within the film. The high charge NFC pair (1) shows the largest swelling, followed by the low charge NFC pair (3), whereas the smallest swelling was observed for the crosslinked high charge NFC pair (2). Crosslinking clearly reduces the moisture induced film swelling by $10-15 \%$.

The dynamic swelling and deswelling curves are almost exponential, showing a fast initial response within the order of seconds, followed by a slower response reaching its limiting value $\sim 20 \mathrm{~min}$ later. It is likely that the slow response shows a relaxation process within the LbL films as it is adjusting its interactions to include more water molecules. The noncrosslinked 48 bilayers containing high charge NFC shows an unexpected decreased swelling with time. This is probably caused by unwanted leakage of low humidity air into the measurement chamber.
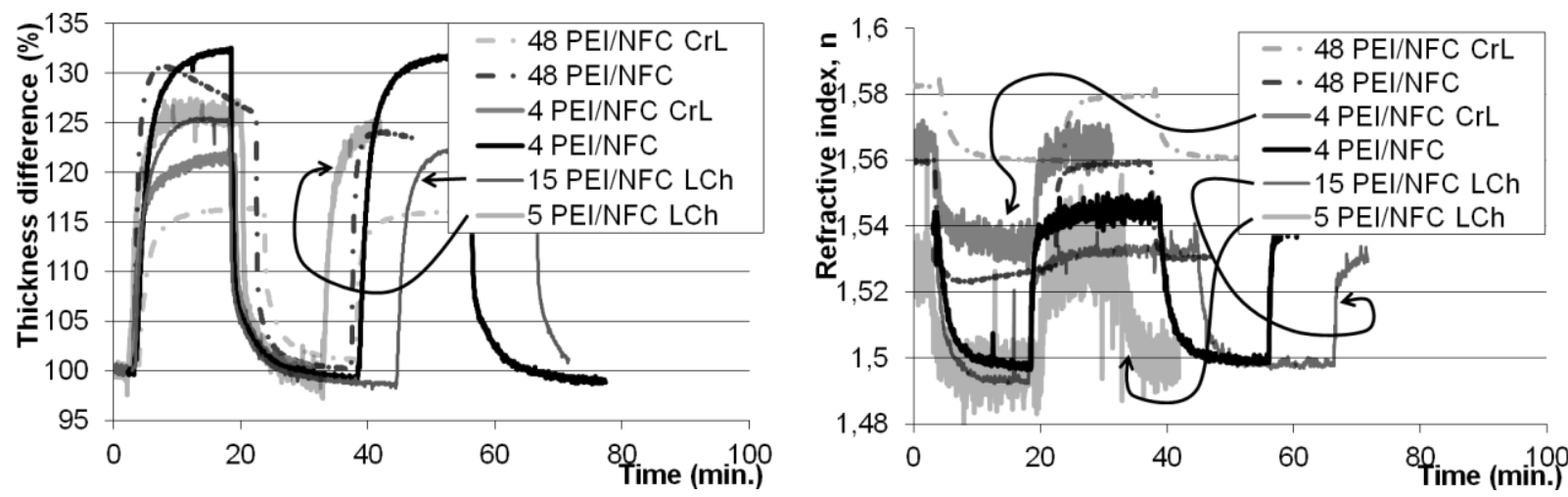

Fig 1. Dynamic relative thickness (left) and refractive index at $600 \mathrm{~nm}$ (right) during two exposures to nitrogen gas $\mathrm{RH}=0 \%$ ( $<3$ min \& $\sim 20<\mathrm{t}<\sim 45 \mathrm{~min})$ and $84-88 \% \mathrm{RH}(3<t \sim 20 \mathrm{~min} \& \sim 35<t<\sim 70 \mathrm{~min})$ followed by a second moisture exposure after 18-25 min. The LbL films are assumed to be homogeneous and isotropic. $\mathrm{CrL}=$ crosslinked, $\mathrm{LCh}=$ low charge. 

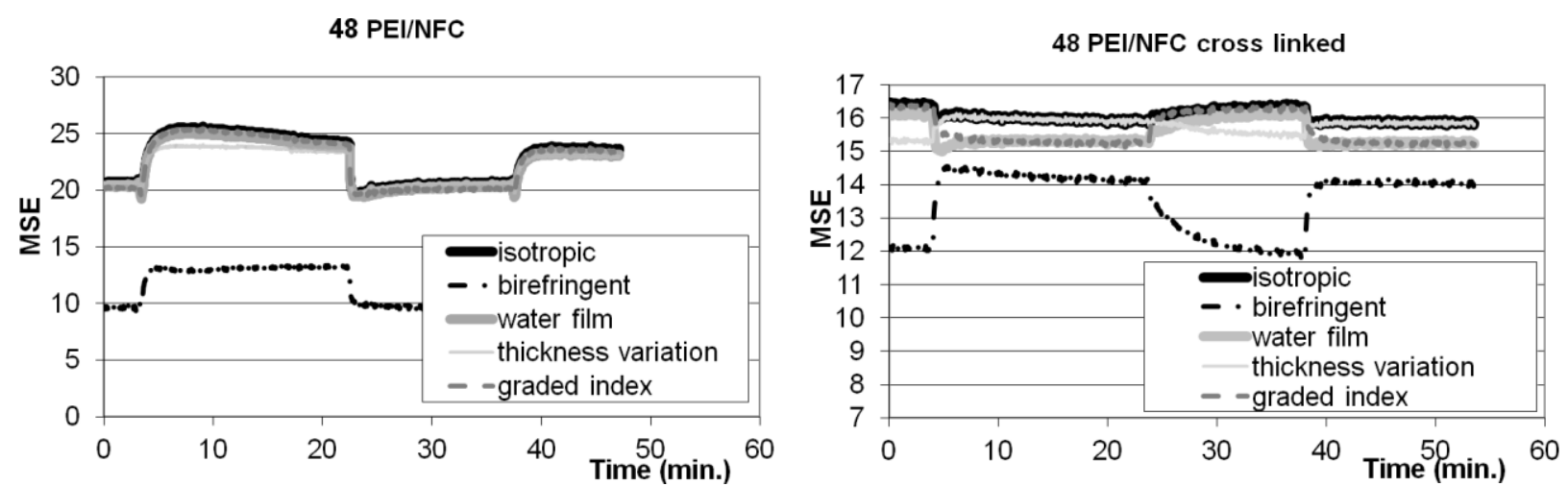

Fig 2 Mean square error (MSE) versus time of the isotropic, birefringent, water film on top, thickness variation, and graded refractive index models for the noncrosslinked and crosslinked $48 \mathrm{PEI} / \mathrm{NFC}$ films. The film was exposed to nitrogen gas $\mathrm{RH}=0 \%(\mathrm{t}<3 \& 23<\mathrm{t}<37$ $\min )$ and $84-88 \% \mathrm{RH}(3<\mathrm{t}<23 \& 37+\mathrm{min})$.
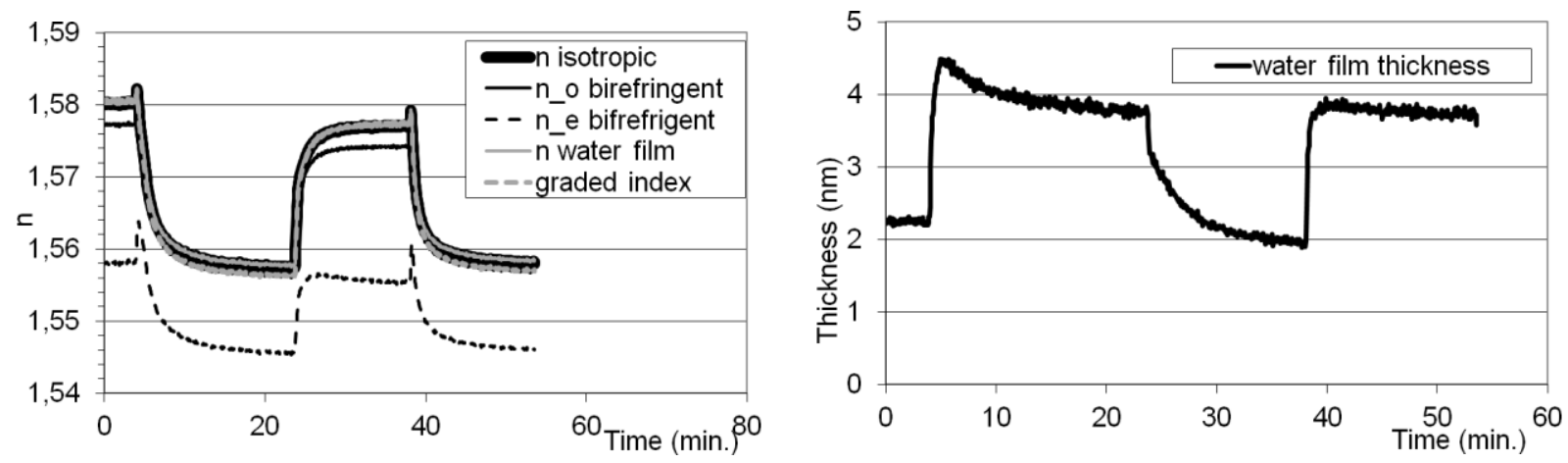

Fig 3 Results of complex models (birefringent, water film on top), compared to a simple isotropic model of the 48 layers of crosslinked PEI/NFC film. The film was exposed to nitrogen gas $\mathrm{RH}=0 \%$ ( $\mathrm{t}<3$ \& $23<\mathrm{t}<37 \mathrm{~min}$ ) and $84-88 \% \mathrm{RH}(3<\mathrm{t}<23$ \& $37+\mathrm{min})$. (left) Refractive index at $600 \mathrm{~nm}$. (right) Thickness of the condensed water film on top of the LbL film.

Fig 1 (right) shows that the refractive indices of the films decrease as the water penetrates into the film. This can be understood as an intermixing of the low refractive index of water with that of the dry LbL film $\left(n_{\text {water }} \approx 1.33\right.$ is smaller than $1.52<n_{\text {dry LbL }}<1.59$ ).

Fig 2 shows the MSE for the different optical models, where a lower number indicates a better fit to measurements. The water film, the graded index, and the thickness variation models give results similar to those obtained with the simpler isotropic model, whereas the birefringent model shows considerable improvement, reducing the MSE by $50 \%$ and $10-25 \%$ for the noncrosslinked and crosslinked $48 \mathrm{PEI} / \mathrm{NFC}$ films, respectively. These results indicate that the water film, the graded index, and the thickness variation models do not explain our measurements much better than the isotropic model despite the addition of one extra free variable. However, the improved fit for the birefringent model indicates that the samples are birefringent and comprise an anisotropic structure. This can be expected since the high aspect ratio of the NFC particles strongly favors orientation along the plane of the film rather than normal to the surface. The absence of a valid graded index model indicates that the films swell uniformly in the presence of humid air. The obtained birefringent model parameters are presented in Table 1.

A closer look at the resulting dynamic refractive index of the 48 layers of crosslinked PEI/NFC (Fig 3, left)
Table 1. Parameter fit (birefringent model) to ellipsometry data at room temperature and $20 \%<\mathrm{RH}<28 \%$. CrL=crosslinked, LCh=low charge, Ao=ordinary, Ae=extraordinary.

\begin{tabular}{llllll}
\hline Sample & Ao & Ae & $\mathbf{B}^{*} \mathbf{1 0}^{-3}$ & $\mathbf{k}$ & $\mathbf{t}[\mathbf{n m}]$ \\
\hline 48 PEI/NFC CrL & 1.552 & 1.531 & 8.68 & 0.030 & 239 \\
48 PEI/NFC & 1.540 & 1.518 & 5.46 & 0.000 & 307 \\
4 PEI/NFC CrL & 1.570 & 1.394 & 4.27 & 0.017 & 19 \\
4 PEI/NFC & 1.527 & 1.427 & 2.65 & 0.000 & 30 \\
15 PEI/NFC LCh & 1.526 & 1.402 & 0.57 & 0.000 & 28 \\
5 PEI/NFC LCh & 1.502 & 1.403 & 6.39 & 0.000 & 15 \\
\hline
\end{tabular}

reveals a small peak during the first 3-4 min after the humid air has entered the measurement chamber. All LbL samples in this study show a similar behavior. This indicates that the water condensates on the surface, filling some surface pores $\left(n_{\text {water }} \approx 1.33>n_{\text {pores }}=1\right)$ or adds a separate water layer on top of the LbL structure. Since the merit of modeling a separate water layer on top of the LbL structure is low and the obtained thickness of the water layer was found to be negative (not physically interpretable) for all samples except for one showing a very thin positive layer of water (Fig 3, right), it can be assumed that there is no separate water film on top of the films. In addition, such a film does not explain the small peak in the refractive index since it still shows up in the left figure. In the birefringent model, the small water condensation peak is larger for the extraordinary 
(perpendicular to the surface) than for the ordinary refractive index of the film. The results (Fig 3, left) also show that the swelling of the film due to inclusion of water dominantly affects the ordinary refractive index, as the extraordinary refractive index reduction is $\approx 60 \%$ of the reduction of the ordinary refractive index.

\section{Conclusions}

This study has used different optical models to describe ellipsometry measurements data of interference films and how the film properties alter in dry and humid environments. The films were prepared with the Layerby-Layer method and contained polyethyleneimine (PEI) and nanofibrillated cellulose (NFC) on silicon wafers.

It was found that the swelling ratio is almost independent of the number of layers within the LbL film and hence also its thickness. The swelling ratio can be affected by the charge density of the NFC (higher charge $\rightarrow$ larger swelling) and can be reduced by heat treatment crosslinking. The results indicate that water condensation initially is filling the surface pores within seconds whereas relaxation of the film to adjust to the added water is a slower process that reaches a steady state after $\approx 20 \mathrm{~min}$. No separate water layer was present on the LbL films. The absence of a valid graded index model indicates that the films swell uniformly in the thickness direction in the presence of humid air.

The films showed a clear birefringence that must be modeled in order to describe the angle-dependent colour of the interference films in detail. Additionally, since the film swelling dominantly affected the ordinary refractive index we conclude that the film swelling ratio is different along the plane of the film compared to the direction normal to the surface. These results provide important understanding for the design of NFC based LbL films for visual moisture sensors and interactive security paper.

\section{Acknowledgements}

We would like to thank the partners within the Paper Beacon project, Vinnova, and RISE for financial support and the possibility to share the results. Knut and Alice Wallenberg are acknowledged for support to instrumentation.

\section{Literature}

Azzam, R. M. A. and Bashara, N. M. (1986): Ellipsometry and Polarized Light, North-Holland.

Burke, S.E. and Barrett, C.J. (2005): Swelling Behavior of Hyaluronic Acid/Polyallylamine Hydrochloride Multilayer Films, Biomacromolecules, 6(5), 1419-1428.

Decher, G. (1997): Fuzzy Nanoassemblies: Toward Layered Polymeric Multicomposites, Science, 277(5330), 1232-1237.

Decher, G. and Hong, J.D. (1991): Buildup of Ultrathin Multilayer Films by a Self-Assembly Process: II. Consecutive Adsorption of Anionic and Cationic Bipolar Amphiphiles and Polyelectrolytes on Charged Surfaces, Berichte Der BunsenGesellschaft-Physical Chemistry Chemical Physics, 95(11), 1430-1434.
Eichhorn, S. J., Dufresne, A., Aranguren, M., Marcovich, N. E., Capadona, J. R., Rowan, S. J., Weder, C., Thielemans, W., Roman, M., Renneckar, S., Gindl, W., Veigel, S., Keckes, J., Yano, H., Abe, K., Nogi, M., Nakagaito, A. N., Mangalam, A., Simonsen, J., Benight, A. S., Bismarck, A., Berglund, L. A. and Peijs, T. (2010): Review: current international research into cellulose nanofibres and nanocomposites, Journal of Materials Science, 45(1), 1-33.

Granberg, H., Forsberg, S., Wågberg L. (2008): Preparation of opto-active materials from microfibrillated cellulose, Cell 224, 235th ACS National Meeting, LA.

Herzinger, C. M., Johs, B., McGahan, W. A., Woollam, J. A., Paulson, W. (1998): Ellipsometric determination of optical constants for silicon and thermally grown silicon dioxide via a multi-sample, multi-wavelength, multi-angle investigation, Journal of Applied Physics, 83(6), 3323-3236.

Klemm, D., Kramer, F., Moritz, S., Lindström, T., Ankerfors, M., Gray, D. and Dorris, A. (2011): Nanocelluloses: A New Family of Nature-Based Materials, Angew. Chem. Int. Ed., 50(24), 2-31.

Levenberg, K. (1944): A Method for the Solution of Certain Non-Linear Problems in Least Squares, The Quarterly of Applied Mathematics, 2, 164-168.

Lösche, M., Schmitt, J., Decher, G., Bouwman, W. G., Kjaer, K. (1998): Detailed Structure of Molecularly Thin Polyelectrolyte Multilayer Films on Solid Substrates as Revealed by Neutron Reflectometry, Macromolecules, 31(25), 8893-8906.

Nolte, A. J., Treat, N. D., Cohen, R. E., Rubner, M. F. (2008): Effect of Relative Humidity on the Young's Modulus of Polyelectrolyte Multilayer Films and Related Nonionic Polymers, Macromolecules, 41(15), 5793-5798.

Secrist, K.E. and Nolte, A.J. (2011): Humidity Swelling/Deswelling Hysteresis in a Polyelectrolyte Multilayer Film, Macromolecules, 44(8), 2859-2865.

Tanchak, O.M. and Barrett, C.J. (2004): Swelling Dynamics of Multilayer Films of Weak Polyelectrolytes, Chem. Mater. 16(14), 2734-2739.

Wågberg, L., Decher, G., Norgren, M., Lindström, T., Ankerfors, M. and Axnäs, K. (2008): The Build-Up of Polyelectrolyte Multilayers of Microfibrillated Cellulose and Cationic Polyelectrolytes, Langmuir 24(3), 784-795.

Wong, J.E., Rehfeldt, F., Hänni, P., Tanaka, M. and Klitzing, R. (2004): Swelling Behavior of Polyelectrolyte Multilayers in Saturated Water Vapor, Macromolecules, 37(19), 7285-7289. 